\title{
Test yourself: right knee pain after fall
}

\author{
Jau-Ann Tsai ${ }^{1} \cdot$ Kush Shah $^{1} \cdot$ A. Ross Sussman ${ }^{1,2}$ \\ Published online: 6 July 2020 \\ (C) ISS 2020
}

\section{Discussion}

This 12-year-old presented after hitting her right knee during a fall on a cruise ship. MRI was performed 2 weeks after the injury and demonstrated exuberant bone marrow edema throughout the proximal tibial metaphysis and epiphysis in the setting of a Salter-Harris injury. On all pulse sequences, abnormal low linear signal was observed within the posterior half of the tibial growth plate, representing trapped periosteum (Figs. 1, 2, and 3). There is also fluid along the growth plate in the setting of a Salter-Harris injury (Fig. 4). Additionally, the MCL was torn distally (Fig. 4).

Trapped periosteum is a complication that may arise from injury to an open growth plate, and when present usually occurs in association with Salter-Harris I or II injuries [1]. Most commonly, the mechanism of Salter-Harris injury at the proximal tibia is an indirect valgus force [1]; however, trapped periosteum can occur not only at the knee but at any site of physeal injury. Radiographs are often used during initial evaluation, although were not available in our case. Radiographs may demonstrate widening of the portion of the growth plate containing the displaced periosteal tissue. If post-reduction radiography shows persistent growth plate widening of more than $3 \mathrm{~mm}$, the incidence of premature growth plate closure increases to $60 \%$, compared with $17 \%$ in cases without growth plate widening [2]. Although our case focuses on trapped periosteum, other soft tissues can become entrapped within the growth plate as well, including ligament, tendon, muscle, nerve, and vessel $[2,3]$. MRI is needed to

Jau-Ann Tsai, Kush Shah and A. Ross Sussman contributed equally to this work.

Jau-Ann Tsai

jauann.tsai@univrad.com

1 Department of Radiology, Robert Wood Johnson Medical School, New Brunswick, NJ 08901, USA

2 University Radiology Group, 579A Road, East Brunswick, Cranbury, NJ 08816, USA

confirm the nature of the entrapped material [3]. On MR imaging, entrapped periosteum appears as a low signal band within the physis on all pulse sequences [1].

Open reduction is often performed to prevent premature physeal closure [3]. However, open treatment may not always be completely successful. In the report by Chen, et al., for instance, a patient with trapped femoral periosteum who was surgically treated nevertheless developed a 1-cm leg length discrepancy that ultimately required physiodesis of the contralateral extremity [3]. Conversely, Poulsen et al. report a patient who did not develop early growth plate closure despite purely conservative management, via knee immobilizer [4].

In the case we present, the surgeon opted for conservative management instead of open reduction, despite the sizeable flap of periosteal tissue within the physis. The patient was fitted with a knee immobilizer and followed up with the surgeon at regular intervals. She also underwent approximately 10 months of physical therapy. She did not bear weight on her knee for 5 months. Full mobility of the knee was not regained until 1 year after the initial injury. No known follow-up studies were obtained. Although the recovery time was long, this case supports that conservative management of a Salter-Harris fracture complicated by trapped periosteum may sometimes be a valid option.

\section{Compliance with ethical standards}

Conflict of interest The authors declare that they have no conflict of interest.

\section{References}

1. Whan A, et al. MRI of Trapped Periosteum in a Proximal Tibial Physeal Injury of a Pediatric Patient. Am J Roentgenol. 2003;181(5):1397-9. https://doi.org/10.2214/ajr.181.5.1811397.

2. Nguyen JC, et al. Imaging of Pediatric Growth Plate Disturbances. RadioGraphics. 2017;37(6):1791-812. https://doi.org/10.1148/rg. 2017170029.

3. Chen J, et al. Imaging Appearance of Entrapped Periosteum within a Distal Femoral Salter-Harris II Fracture. Skeletal Radiol. 2015;44(10):1547-51. https://doi.org/10.1007/s00256-015-2201-x. 
4. Poulsen DR, et al. Trapped Periosteum Managed Conservatively. Eurorad. 2017;5. https://doi.org/10.1594/EURORAD/CASE.14569.
Publisher's note Springer Nature remains neutral with regard to jurisdictional claims in published maps and institutional affiliations. 